PROCEEDINGS OF THE

AMERICAN MATHEMATICAL SOCIETY

Volume 137, Number 10, October 2009, Pages 3369-3377

S 0002-9939(09)09970-5

Article electronically published on May 21, 2009

\title{
A DIV-CURL DECOMPOSITION FOR THE LOCAL HARDY SPACE
}

\author{
DER-CHEN CHANG, GALIA DAFNI, AND HONG YUE
}

(Communicated by Mei-Chi Shaw)

\begin{abstract}
A decomposition theorem for the local Hardy space of Goldberg, in terms of nonhomogeneous div-curl quantities, is proved via a dual result for the space bmo.
\end{abstract}

\section{INTRODUCTION}

Given vector fields $\mathbf{V}=\left(v_{1}, \ldots, v_{n}\right)$ in $L^{p}\left(\mathbb{R}^{n}, \mathbb{R}^{n}\right)$ and $\mathbf{W}=$ $\left(w_{1}, \ldots, w_{n}\right)$ in $L^{p^{\prime}}\left(\mathbb{R}^{n}, \mathbb{R}^{n}\right)$ with $1<p<\infty, \frac{1}{p}+\frac{1}{p^{\prime}}=1$, the scalar (dot) product $\mathbf{V} \cdot \mathbf{W}=\sum v_{i} w_{i}$ will lie in $L^{1}\left(\mathbb{R}^{n}\right)$. Coifman, Lions, Meyer, and Semmes [CLMS] showed that if the following conditions hold in the sense of distributions:

$$
\begin{gathered}
\operatorname{div} \mathbf{V}:=\sum \frac{\partial v_{i}}{\partial x_{i}}=0, \\
\operatorname{curl} \mathbf{W}:=\left(\frac{\partial w_{j}}{\partial x_{i}}-\frac{\partial w_{i}}{\partial x_{j}}\right)_{i j}=0,
\end{gathered}
$$

then $\mathbf{V} \cdot \mathbf{W}$ belongs to the real Hardy space $H^{1}\left(\mathbb{R}^{n}\right)$, a proper subspace of $L^{1}$. Moreover,

$$
\|\mathbf{V} \cdot \mathbf{W}\|_{H^{1}} \leq C\|\mathbf{V}\|_{L^{p}}\|\mathbf{W}\|_{L^{p^{\prime}}} .
$$

This "div-curl lemma" and other results of a similar nature illustrate the recent use of Hardy spaces for applications to nonlinear PDEs and in the method of compensated compactness, originally going back to the work of Murat and Tartar.

Recall (see [FS]) that a function $f$ belongs to $H^{1}\left(\mathbb{R}^{n}\right)$ if the maximal function $M_{\varphi}(f)$ belongs to $L^{1}\left(\mathbb{R}^{n}\right)$, where, for a fixed Schwartz function $\varphi, \int \varphi=1$, we define

$$
M_{\varphi}(f)(x)=\sup _{t>0}\left|f * \varphi_{t}(x)\right|, \quad \varphi_{t}(\cdot)=t^{-n} \varphi\left(t^{-1} \cdot\right) .
$$

Here the norm, defined by $\|f\|_{H^{1}}:=\left\|M_{\varphi}\right\|_{L^{1}}$, depends on the choice of $\varphi$, but the space does not since different choices of $\varphi$ give equivalent norms. Functions

Received by the editors January 21, 2009.

2000 Mathematics Subject Classification. Primary 42B30; Secondary 35B65, 35F05, 46E35.

The first author was partially supported by a Hong Kong RGC competitive earmarked research grant \#600607 and a competitive research grant at Georgetown University.

The second author was partially supported by the Natural Sciences and Engineering Research Council, Canada.

The third author was partially supported by the Natural Sciences and Engineering Research Council, Canada, and the Centre de Recherches Mathématiques, Montreal. 
in the Hardy space enjoy both improved integrability and cancellation conditions compared to $L^{1}$ functions. In particular, if $f \in H^{1}$, then its integral must vanish.

The local real Hardy space $h^{1}\left(\mathbb{R}^{n}\right)$, defined by Goldberg [Go, is larger than $H^{1}$ and allows for more flexibility, since global cancellation conditions are not necessary. For example, the Schwartz space is contained in $h^{1}$ but not in $H^{1}$, and multiplication by cutoff functions preserves $h^{1}$ but not $H^{1}$, thus making it more suitable for working in domains and on manifolds. For membership of a function $f$ in $h^{1}\left(\mathbb{R}^{n}\right)$, we use a "local" maximal function $m_{\varphi}(f)$, with

$$
m_{\varphi}(f)(x)=\sup _{0<t<1}\left|f * \varphi_{t}(x)\right|,
$$

and require $m_{\varphi}(f) \in L^{1}\left(\mathbb{R}^{n}\right)$. As for $H^{1},\|f\|_{h^{1}}:=\left\|m_{\varphi}\right\|_{L^{1}}$ defines a norm, and different choices of $\varphi$ give equivalent norms, so we can choose $\varphi$ with compact support, making clear the local nature of the maximal function.

In $\mathrm{D}$, sufficient nonhomogeneous conditions on the divergence and curl were found in order for the dot product $\mathbf{V} \cdot \mathbf{W}$, where $\mathbf{V}$ and $\mathbf{W}$ are vector fields as above, to be in $h^{1}\left(\mathbb{R}^{n}\right)$. In particular, as a corollary, the following special case was proved:

Proposition $1.1([\mathrm{D}])$. Suppose $\mathbf{V}$ and $\mathbf{W}$ are vector fields on $\mathbb{R}^{n}$ satisfying

$$
\mathbf{V} \in L^{p}\left(\mathbb{R}^{n}\right)^{n}, \quad \mathbf{W} \in L^{p^{\prime}}\left(\mathbb{R}^{n}\right)^{n}, \quad 1<p<\infty, \quad \frac{1}{p}+\frac{1}{p^{\prime}}=1 .
$$

If there exists a function $f$ in $L^{p}\left(\mathbb{R}^{n}\right)$ and a matrix-valued function $A$ with components in $L^{p^{\prime}}\left(\mathbb{R}^{n}\right)$ such that, in the sense of distributions,

$$
\operatorname{div} \mathbf{V}=f, \quad \operatorname{curl} \mathbf{W}=A,
$$

then $\mathbf{V} \cdot \mathbf{W}$ belongs to the local Hardy space $h^{1}\left(\mathbb{R}^{n}\right)$ with

$$
\|\mathbf{V} \cdot \mathbf{W}\|_{h^{1}} \leq C\left(\|\mathbf{V}\|_{L^{p}}\|\mathbf{W}\|_{L^{p^{\prime}}}+\|f\|_{L^{p}}\|\mathbf{W}\|_{L^{p^{\prime}}}+\|\mathbf{V}\|_{L^{p}} \sum_{i, j}\left\|A_{i j}\right\|_{L^{p^{\prime}}}\right) .
$$

Note that the requirement on the divergence and the components of the curl to be functions in $L^{p}$ and $L^{p^{\prime}}$, respectively, was also used in the original div-curl lemma of Murat $[\mathrm{Mu}$ and is a natural relaxation of the vanishing divergence and curl conditions. In fact, by the Hodge decomposition, this can be viewed as a combination of the following two theorems:

Theorem $1.2([\mathrm{D}])$. Suppose $\mathbf{V}$ and $\mathbf{W}$ are vector fields on $\mathbb{R}^{n}$ satisfying

$$
\mathbf{V} \in L^{p}\left(\mathbb{R}^{n}\right)^{n}, \quad \mathbf{W} \in L^{p^{\prime}}\left(\mathbb{R}^{n}\right)^{n}, \quad 1<p<\infty, \quad \frac{1}{p}+\frac{1}{p^{\prime}}=1
$$

and

$$
\operatorname{div} \mathbf{V}=f \in L^{p}\left(\mathbb{R}^{n}\right), \quad \operatorname{curl} \mathbf{W}=0
$$

in the sense of distributions. Then $\mathbf{V} \cdot \mathbf{W}$ belongs to the local Hardy space $h^{1}\left(\mathbb{R}^{n}\right)$ with

$$
\|\mathbf{V} \cdot \mathbf{W}\|_{h^{1}\left(\mathbb{R}^{n}\right)} \leq C\left(\|\mathbf{V}\|_{L^{p}\left(\mathbb{R}^{n}\right)}+\|f\|_{L^{p}\left(\mathbb{R}^{n}\right)}\right)\|\mathbf{W}\|_{L^{p^{\prime}}\left(\mathbb{R}^{n}\right)} ;
$$

and 
Theorem $1.3\left([\mathrm{D})\right.$. Suppose $\mathbf{V}$ and $\mathbf{W}$ are vector fields on $\mathbb{R}^{n}$ satisfying

$$
\mathbf{V} \in L^{p}\left(\mathbb{R}^{n}\right)^{n}, \quad \mathbf{W} \in L^{p^{\prime}}\left(\mathbb{R}^{n}\right)^{n}, \quad 1<p<\infty, \quad \frac{1}{p}+\frac{1}{p^{\prime}}=1 .
$$

If $A$ is a matrix with components in $L^{p^{\prime}}\left(\mathbb{R}^{n}\right)$ and if

$$
\operatorname{div} \mathbf{V}=0, \quad \operatorname{curl} \mathbf{W}=A
$$

in the sense of distributions, then $\mathbf{V} \cdot \mathbf{W}$ belongs to the local Hardy space $h^{1}\left(\mathbb{R}^{n}\right)$ with

$$
\|\mathbf{V} \cdot \mathbf{W}\|_{h^{1}\left(\mathbb{R}^{n}\right)} \leq C\|\mathbf{V}\|_{L^{p}\left(\mathbb{R}^{n}\right)}\left[\|\mathbf{W}\|_{L^{p^{\prime}\left(\mathbb{R}^{n}\right)}}+\sum_{i, j}\left\|A_{i j}\right\|_{L^{p^{\prime}}\left(\mathbb{R}^{n}\right)}\right] .
$$

It may seem that the conditions on the divergence and the curl in the theorems above are too strong, so the question arises as to whether one can characterize functions in the Hardy space in terms of such div-curl quantities. Such a characterization, providing a kind of converse to the div-curl lemma, was shown in [CLMS] for $H^{1}\left(\mathbb{R}^{n}\right)$ :

Theorem 1.4 ([CLMS $)$. Every function $f \in H^{1}\left(\mathbb{R}^{n}\right)$ can be written as

$$
f=\sum_{k=1}^{\infty} \lambda_{k} \mathbf{V}_{k} \cdot \mathbf{W}_{k}
$$

with $\left\{\lambda_{k}\right\} \in \ell^{1}$ and $\mathbf{V}_{k}, \mathbf{W}_{k}$ vector fields with norm bounded by 1 in $L^{2}\left(\mathbb{R}^{n}, \mathbb{R}^{n}\right)$, and $\operatorname{div} \mathbf{V}_{k}=0$, curl $\mathbf{W}_{k}=0$ in the sense of distributions on $\mathbb{R}^{n}$.

This decomposition was proved in [CLMS, via functional analysis arguments, from the following dual result: for $g \in \operatorname{BMO}\left(\mathbb{R}^{n}\right)$,

$$
\|g\|_{\mathrm{BMO}} \approx \sup _{\mathbf{V}, \mathbf{W}} \int_{\mathbb{R}^{n}} g \mathbf{V} \cdot \mathbf{W}
$$

where the supremum is taken over all vector fields $\mathbf{V}, \mathbf{W}$ in $L^{2}\left(\mathbb{R}^{n}, \mathbb{R}^{n}\right)$, $\|\mathbf{V}\|_{L^{2}},\|\mathbf{W}\|_{L^{2}} \leq 1$, satisfying $\operatorname{div} \mathbf{V}=0$, curl $\mathbf{W}=0$ in the sense of distributions on $\mathbb{R}^{n}$.

The goal of this paper is to prove an analogue, Theorem 2.2, of (1.4) for functions in $\operatorname{bmo}\left(\mathbb{R}^{n}\right)$, the dual of the local Hardy space $h^{1}\left(\mathbb{R}^{n}\right)$, and consequently a decomposition theorem, Theorem 3.1 for $h^{1}$ in terms of the div-curl quantities used in Theorem 1.2 or in Theorem 1.3 .

\section{The DIV-CURL LEMMA FOR LOCAL BMO}

In [Go it was shown that the dual of $h^{1}\left(\mathbb{R}^{n}\right)$ can be identified with the space $\operatorname{bmo}\left(\mathbb{R}^{n}\right)$, consisting of locally integrable functions $f$ with

$$
\|f\|_{\mathrm{bmo}}:=\sup _{|I| \leq 1} \frac{1}{|I|} \int_{I}\left|f-f_{I}\right|+\sup _{|I|>1} \frac{1}{|I|} \int_{I}|f|<\infty .
$$

Here the supremum can be taken over balls or cubes with sides parallel to the axes, $|I|$ denotes Lebesgue measure (volume) and $f_{I}$ is the mean of $f$ over $I$, i.e. $\frac{1}{|I|} \int_{I} f$. The number 1 in the definition can be replaced by any other finite nonzero constant. Note that unlike the case of BMO, we do not need to consider this norm modulo constants.

Before stating the main result, let us introduce the following definition in order to simplify notation. 
Definition 2.1. Denote by $\mathcal{D} \mathcal{C}_{1,0}^{p}$ the collection of all functions which can be written in the form $\mathbf{V} \cdot \mathbf{W}$, where $\mathbf{V}, \mathbf{W}$ are vector fields, $\mathbf{V} \in L^{p}\left(\mathbb{R}^{n}, \mathbb{R}^{n}\right), \mathbf{W} \in$ $L^{p^{\prime}}\left(\mathbb{R}^{n}, \mathbb{R}^{n}\right),\|\mathbf{V}\|_{L^{p}} \leq 1,\|\mathbf{W}\|_{L^{p^{\prime}}} \leq 1$, satisfying, in the sense of distributions on $\mathbb{R}^{n}$

$$
\operatorname{div} \mathbf{V}=f \in L^{p}\left(\mathbb{R}^{n}\right), \quad\|f\|_{L^{p}} \leq 1, \quad \text { and } \quad \operatorname{curl} \mathbf{W}=0 .
$$

The collection $\mathcal{D C}_{0,1}^{p}$ can be defined analogously by requiring the vector fields to satisfy, instead of (2.1), the conditions

$$
\operatorname{div} \mathbf{V}=0,(\operatorname{curl} \mathbf{W})_{i j}=A_{i j} \in L^{p^{\prime}}\left(\mathbb{R}^{n}\right),\left\|A_{i j}\right\|_{L^{p^{\prime}}} \leq 1, i, j \in\{1, \ldots, n\} .
$$

Note that by Theorems 1.2 and 1.3 , both $\mathcal{D} \mathcal{C}_{1,0}^{p}$ and $\mathcal{D C} \mathcal{C}_{0,1}^{p}$ are subsets of $h^{1}\left(\mathbb{R}^{n}\right)$ for every $1<p<\infty$.

Theorem 2.2. If $g \in \operatorname{bmo}\left(\mathbb{R}^{n}\right)$, then for $1<p<\infty$,

$$
\|g\|_{\mathrm{bmo}} \approx \sup _{f \in \mathcal{D} \mathcal{C}_{1,0}^{p}} \int_{\mathbb{R}^{n}} g f
$$

and

$$
\|g\|_{\mathrm{bmo}} \approx \sup _{f \in \mathcal{D} \mathcal{C}_{0,1}^{p}} \int_{\mathbb{R}^{n}} g f
$$

with constants that depend only on $p$ and the dimension.

Proof. Let $g \in \operatorname{bmo}\left(\mathbb{R}^{n}\right.$ ) (we are assuming that $g$ is real-valued) and take $f \in$ $\mathcal{D C}_{1,0}^{p} \cup \mathcal{D} \mathcal{C}_{0,1}^{p}$. As stated above, by Theorems 1.2 and $1.3 f$ belongs to $h^{1}\left(\mathbb{R}^{n}\right)$ with norm bounded by a constant. The duality of $\operatorname{bmo}\left(\mathbb{R}^{n}\right)$ with $h^{1}\left(\mathbb{R}^{n}\right)$ then gives

$$
\int_{\mathbb{R}^{n}} g f \leq C\|g\|_{\mathrm{bmo}} .
$$

It remains to prove the other direction, i.e.

$$
\|g\|_{\text {bmo }} \leq C^{\prime} \int_{\mathbb{R}^{n}} g f,
$$

where we take the supremum over $f \in \mathcal{D} \mathcal{C}_{1,0}^{p}$ or $f \in \mathcal{D} \mathcal{C}_{0,1}^{p}$, respectively.

We will use the definition of bmo with the supremum taken over balls.

Case I: If $B$ is a small ball, say with radius bounded by 1 (although in fact the proof is independent of the radius), one can use the following estimate from the proof of Theorem III.2 in CLMS:

$$
\left(\frac{1}{|B|} \int_{B}\left|g(x)-g_{B}\right|^{2} d x\right)^{1 / 2} \leq C_{n} \sup \int g \mathbf{V} \cdot \mathbf{W},
$$

where the supremum is taken over all vector fields $\mathbf{V}, \mathbf{W} \in C_{0}^{\infty}(\widetilde{B})$ (here and below $\widetilde{B}$ denotes the ball with the same center and twice the radius of $B$ ) with $\|\mathbf{V}\|_{L^{p}} \leq 1,\|\mathbf{W}\|_{L^{p^{\prime}}} \leq 1$ and $\operatorname{div} \mathbf{V}=0, \operatorname{curl} \mathbf{W}=0$. Note that the result in CLMS is stated for a cube $Q$ (instead of a ball $B$ ) and for $p=p^{\prime}=2$, but can be modified to the case of a ball and for $p \neq 2$ as follows.

The key inequality used in the proof in CLMS,

$$
\left\|g-g_{B}\right\|_{L^{2}(B)} \leq C \sum_{i=1}^{n}\left\|\frac{\partial g}{\partial x_{i}}\right\|_{W^{-1,2}(B)},
$$


is valid for any Lipschitz domain (see for example [GR, Section I.2.1, Corollary 2.1). This inequality holds in the homogeneous sense, modulo constants. Therefore, while we denote by $W^{-1,2}(B)$ the dual of the Sobolev space $W_{0}^{1,2}(B)$, which is the closure of $C_{0}^{\infty}(B)$ under the norm $\|\varphi\|_{W^{1,2}(B)}=\|\varphi\|_{L^{2}(B)}+\|\nabla \varphi\|_{L^{2}(B)}$, to obtain the righthand side of (2.6) we test against test functions bounded only in the homogeneous Sobolev norm $\|u\|_{\dot{W}^{1,2}(B)}=\|\nabla u\|_{L^{2}(B)}$. For a fixed ball the homogeneous and nonhomogeneous norms on $C_{0}^{\infty}(B)$ functions are equivalent, but here we use only the homogeneous norm in order for the constant to be independent of the radius of the ball.

To estimate $\left\|\frac{\partial g}{\partial x_{i}}\right\|_{W^{-1,2}(B)}, i=1, \ldots, n$, we fix a $j \in\{1, \ldots, n\} \backslash\{i\}$ and define the vector fields $\mathbf{V}$ and $\mathbf{W}$, for the case $p \leq 2$, as in CLMS: given $u \in C_{0}^{\infty}(B)$ with $\|\nabla u\|_{L^{2}} \leq 1$, let

$$
\mathbf{V}=|B|^{1 / 2-1 / p}\left(\frac{\partial u}{\partial x_{i}} \mathbf{e}_{j}-\frac{\partial u}{\partial x_{j}} \mathbf{e}_{i}\right)
$$

where $\mathbf{e}_{i}$ denotes the $i$ th coordinate vector in $\mathbb{R}^{n}$, and let

$$
\mathbf{W}=\gamma|B|^{-1 / p^{\prime}} \nabla\left(\left(x_{j}-x_{j}^{0}\right) \eta\left(\frac{x-x^{0}}{R}\right)\right),
$$

where $x^{0}$ and $R$ are the center and radius of $B$, respectively. Here $\eta$ is a fixed smooth function supported in $B(0,2)$ and identically equal to 1 on $B(0,1)$.

Note that since $p \leq 2$, the support and bound on the $L^{2}$ norm of $\nabla u$ imply $\|\mathbf{V}\|_{L^{p}} \leq 1$, while $\gamma$ can be chosen, depending only on $\eta, n$ and $p$, so that $\|\mathbf{W}\|_{L^{p^{\prime}}} \leq$ 1. This gives us the desired properties for $\mathbf{V}$ and $\mathbf{W}$, and moreover,

$$
\mathbf{V} \cdot \mathbf{W}=\gamma|B|^{-1 / 2} \frac{\partial u}{\partial x_{i}} \text {. }
$$

When $p \geq 2$, we need to change the definitions of $\mathbf{V}$ and $\mathbf{W}$. As above, we take $u \in C_{0}^{\infty}(B)$ with $\|\nabla u\|_{L^{2}} \leq 1$, but now we set

$$
\mathbf{W}=|B|^{1 / 2-1 / p^{\prime}} \nabla u
$$

and (again taking $j \in\{1, \ldots, n\} \backslash\{i\}$ )

$$
\mathbf{V}=\gamma^{\prime}|B|^{-1 / p}\left\{\frac{\partial\left(\eta_{B} x_{j}\right)}{\partial x_{j}} \mathbf{e}_{i}-\frac{\partial\left(\eta_{B} x_{j}\right)}{\partial x_{i}} \mathbf{e}_{j}\right\}
$$

with $\eta_{B}$ defined by $\eta_{B}(x)=\eta\left(\frac{x-x^{0}}{R}\right)$ for $\eta$ as above. Clearly $\operatorname{div} \mathbf{V}=0$ and curl $\mathbf{W}=0$, and by the conditions on $u$, the fact that $p \geq 2$, and the choice of $\gamma^{\prime}$, we can make $\|\mathbf{V}\|_{L^{p}} \leq 1$ and $\|\mathbf{W}\|_{L^{p^{\prime}}} \leq 1$. On $B$, where $\eta_{B}$ is identically equal to 1, we get $\mathbf{V}=\gamma^{\prime}|B|^{-1 / p} \mathbf{e}_{i}$, so that as before

$$
\mathbf{V} \cdot \mathbf{W}=\gamma^{\prime}|B|^{-1 / 2} \frac{\partial u}{\partial x_{i}}
$$

Integrating against $g$ in (2.9) or (2.12), we can proceed for any $p \in(1, \infty)$. Taking the supremum over all such $u$, we get a bound on $\left\|\frac{\partial g}{\partial x_{i}}\right\|_{W^{-1,2}(B)}$ by a constant multiple of $|B|^{1 / 2}$ times the right-hand side of (2.5). We then obtain (2.5) from (2.6).

Case II: Now let us consider a large ball $B$ with radius greater than 1 . For this type of ball we need to show a stronger condition; that is, we need to bound the 
mean of $|g|$ on $B$. We will do this by showing two cases of the following inequality, corresponding to (2.3) and (2.4):

$$
\left(\frac{1}{|B|} \int_{B}|g(x)|^{r} d x\right)^{1 / r} \leq C_{n, r} \sup \int g \mathbf{V} \cdot \mathbf{W} .
$$

In both cases the supremum is to be taken over pairs of smooth vector fields $\mathbf{V}$, $\mathbf{W}$ supported in $\tilde{B}, \mathbf{V} \in\left(L^{p}\right)^{n}, \mathbf{W} \in\left(L^{p^{\prime}}\right)^{n}$, with norms bounded by 1 , and in the first case, with $r=p^{\prime}$, the vector fields satisfy condition (2.1), while in the second case, with $r=p$, they satisfy (2.2).

Note that a priori we have the finiteness of the left-hand side of (2.13) by the fact that $g \in$ bmo $\subset \mathrm{BMO} \subset L_{\text {loc }}^{r}$ for any $r<\infty$.

We first prove estimate (2.13) when $B=B_{1}$ is the unit ball $B(0,1)$. In order to do this we need to use the full (nonhomogeneous) version of inequality (2.6), namely

$$
\|g\|_{L^{r}\left(B_{1}\right)} \leq C\left\{\|g\|_{W^{-1, r}\left(B_{1}\right)}+\sum_{i=1}^{n}\left\|\frac{\partial g}{\partial x_{i}}\right\|_{W^{-1, r}\left(B_{1}\right)}\right\}
$$

for any $r, 1<r<\infty$ (see $\mathbb{\mathrm { Ne }}$, Theorem 1, p. 108, with $l=0$ ).

The estimates for $\left\|\frac{\partial g}{\partial x_{i}}\right\|_{W^{-1, r}\left(B_{1}\right)}$ are analogous to those above (for a fixed ball the norm is equivalent to the homogeneous case). Here the test functions are in $W_{0}^{1, r^{\prime}}\left(B_{1}\right)$, so $r^{\prime}$ plays the role of the exponent 2 in the argument above, and again we need to distinguish the two cases. For $r=p^{\prime}$, given $u \in C_{0}^{\infty}(B)$ with $\|\nabla u\|_{L^{p}} \leq 1$, we use the same vector fields as defined in (2.7) and (2.8), normalized for the unit ball $B_{1}$. For $r=p$ the test function $u$ will now have $\|\nabla u\|_{L^{p^{\prime}}} \leq 1$, so we can define the vector fields as in (2.11) and (2.10).

Now we address the part that is different from the homogeneous case: we have to bound

$$
\|g\|_{W^{-1, r}\left(B_{1}\right)}=\sup \left|\int g \varphi\right|,
$$

where the supremum is taken over $\varphi \in C_{0}^{\infty}\left(B_{1}\right),\|\varphi\|_{W^{1, r^{\prime}}\left(B_{1}\right)} \leq 1$. We need to be able to write any such function $\varphi$ in terms of div-curl quantities $\mathbf{V} \cdot \mathbf{W}$.

Lemma 2.3. If $\varphi \in C_{0}^{\infty}\left(B_{1}\right)$, then we can write

$$
\varphi=C \mathbf{V}_{1} \cdot \mathbf{W}_{1}=C \mathbf{V}_{2} \cdot \mathbf{W}_{2}
$$

for smooth vector fields $\mathbf{V}_{i}, \mathbf{W}_{i}$ with $\mathbf{V}_{1}$ and $\mathbf{W}_{2}$ supported in $B_{1}, \mathbf{W}_{1}$ and $\mathbf{V}_{2}$ supported in the double ball $\widetilde{B_{1}}=B(0,2), \mathbf{W}_{1} \in\left(L^{p^{\prime}}\left(\widetilde{B_{1}}\right)\right)^{n}, \mathbf{V}_{2} \in\left(L^{p}\left(\widetilde{B_{1}}\right)\right)^{n}$ (with norms bounded by a constant independent of $\varphi)$, and satisfying

$$
\operatorname{div} \mathbf{V}_{2}=0, \operatorname{curl} \mathbf{W}_{1}=0 .
$$

In addition, we have

(i) $\mathbf{V}_{1} \in\left(L^{p}\left(B_{1}\right)\right)^{n}$ and $\operatorname{div} \mathbf{V}_{1} \in L^{p}\left(B_{1}\right)$ with bounds

$$
\left\|\mathbf{V}_{1}\right\|_{L^{p}} \leq C\|\varphi\|_{L^{p}}, \quad\left\|\operatorname{div} \mathbf{V}_{1}\right\|_{L^{p}} \leq C\|\nabla \varphi\|_{L^{p}} ;
$$

(ii) $\mathbf{W}_{2} \in\left(L^{p^{\prime}}\left(B_{1}\right)\right)^{n}$ and, for all $i, j,\left(\operatorname{curl} \mathbf{W}_{2}\right)_{i j} \in L^{p^{\prime}}\left(B_{1}\right)$ with bounds

$$
\left\|\mathbf{W}_{2}\right\|_{L^{p^{\prime}}} \leq C\|\varphi\|_{L^{p^{\prime}}}, \quad\left\|\left(\operatorname{curl} \mathbf{W}_{2}\right)_{i j}\right\|_{L^{p^{\prime}}} \leq C\|\nabla \varphi\|_{L^{p^{\prime}}} .
$$


Proof of Lemma 2.3. Let $\varphi \in C_{0}^{\infty}\left(B_{1}\right)$. Take

$$
\mathbf{V}_{1}=\varphi \mathbf{e}_{1}=(\varphi, 0, \ldots, 0)
$$

and

$$
\mathbf{W}_{1}=\nabla\left(\eta x_{1}\right)
$$

where $\eta$ is supported in $\widetilde{B_{1}}=B(0,2)$, satisfies $\|\eta\|_{L^{\infty}} \leq 1,\|\nabla \eta\|_{L^{\infty}} \leq 1$, and is identically equal to 1 on the support of $\varphi$. Note that this ensures that on $B_{1}$, $\mathbf{W}_{1}=\mathbf{e}_{1}$, so we get the desired identity

$$
\mathbf{V}_{1} \cdot \mathbf{W}_{1}=\varphi
$$

We can bound the norm of $\mathbf{W}_{1}$ by a constant:

$$
\left\|\mathbf{W}_{1}\right\|_{L^{p^{\prime}}\left(B_{1}\right)} \leq\|\nabla \eta\|_{\infty}\left(\int_{\widetilde{B_{1} \backslash B_{1}}}\left|x_{1}\right|^{p^{\prime}}\right)^{1 / p^{\prime}}+\|\eta\|_{\infty}\left|B_{1}\right|^{1 / p^{\prime}} \leq C_{n, p},
$$

and since $\mathbf{W}_{1}$ is a gradient, we also have curl $\mathbf{W}_{1}=0$.

Moreover

$$
\left\|\mathbf{V}_{1}\right\|_{L^{p}\left(B_{1}\right)} \leq\|\varphi\|_{L^{p}\left(B_{1}\right)}
$$

and

$$
\left\|\operatorname{div} \mathbf{V}_{1}\right\|_{L^{p}\left(B_{1}\right)}=\left\|\frac{\partial \varphi}{\partial x_{1}}\right\|_{L^{p}\left(B_{1}\right)} \leq\|\nabla \varphi\|_{L^{p}\left(B_{1}\right)} .
$$

For the other case, we can take

$$
\mathbf{V}_{2}=\frac{\partial\left(\eta x_{1}\right)}{\partial x_{1}} \mathbf{e}_{2}-\frac{\partial\left(\eta x_{1}\right)}{\partial x_{2}} \mathbf{e}_{1}=\left(-\frac{\partial \eta}{\partial x_{2}}, \frac{\partial \eta}{\partial x_{1}}+1,0, \ldots, 0\right)
$$

with $\eta$ as above. Then

$$
\left\|\mathbf{V}_{2}\right\|_{L^{p}\left(B_{1}\right)} \leq\|\nabla \eta\|_{L^{p}\left(B_{1}\right)} \leq C_{n, p}
$$

and $\operatorname{div} \mathbf{V}_{2}=0$. We also set

$$
\mathbf{W}_{2}=\varphi \mathbf{e}_{2},
$$

so as above, since $\eta$ is identically 1 on the support of $\varphi$, we have

$$
\mathbf{V}_{2} \cdot \mathbf{W}_{2}=\varphi \text {. }
$$

In addition,

and

$$
\left\|\mathbf{W}_{2}\right\|_{L^{p^{\prime}}\left(B_{1}\right)} \leq\|\varphi\|_{L^{p^{\prime}}\left(B_{1}\right)}
$$

$$
\left\|\left(\operatorname{curl} \mathbf{W}_{2}\right)_{i j}\right\|_{L^{p^{\prime}}\left(B_{1}\right)} \leq\|\nabla \varphi\|_{L^{p^{\prime}}\left(B_{1}\right)} .
$$

Continuation of the proof of Theorem 2.2. We obtain estimate (2.13) for the unit ball, with $r=p^{\prime}$ and condition (2.1) satisfied, by using (2.14), applying the lemma with the vector fields $V_{1}$ and $W_{1}$ to the test functions in (2.15), and dividing by the appropriate constants. The case $r=p$ and (2.2) corresponds to using the lemma with $V_{2}$ and $W_{2}$.

Now let us prove estimate (2.13) for a ball $B=B\left(x_{0}, R\right), R \geq 1$. On the left-hand side, any $g \in L^{r}(B)$ corresponds in a one-to-one and onto fashion to a $\tilde{g} \in L^{r}\left(B_{1}\right)$ by taking $\tilde{g}(x)=g\left(x_{0}+R x\right)$, with

$$
\left(\frac{1}{\left|B_{1}\right|} \int_{B_{1}}|\tilde{g}(x)|^{r} d x\right)^{1 / r}=\left(\frac{1}{|B|} \int_{B}|g(y)|^{r} d y\right)^{1 / r} .
$$


On the right-hand side, for any smooth vector fields $\mathbf{V}_{i}, \mathbf{W}_{i}$ corresponding to the unit ball, as in Lemma $2.3\left(i=1\right.$ in the case $r=p^{\prime}, i=2$ in the case $\left.r=p\right)$, define

$$
\mathbf{V}_{0}=\mathbf{V}_{i}\left(R^{-1}\left(x-x_{0}\right)\right), \quad \mathbf{W}_{0}=\mathbf{W}_{i}\left(R^{-1}\left(x-x_{0}\right)\right) .
$$

Then $\mathbf{V}_{0}, \mathbf{W}_{0}$ are smooth vector fields supported in $\widetilde{B}=B\left(x_{0}, 2 R\right), \mathbf{V}_{0} \in\left(L^{p}\right)^{n}$, and $\mathbf{W}_{0} \in\left(L^{p^{\prime}}\right)^{n}$ with bounds

$$
\left\|\mathbf{V}_{0}\right\|_{L^{p}}=\left(\int\left|\mathbf{V}_{i}\left(R^{-1}\left(x-x_{0}\right)\right)\right|^{p} d x\right)^{1 / p}=R^{n / p}\left\|\mathbf{V}_{i}\right\|_{L^{p}} \leq R^{n / p},
$$

and similarly

$$
\left\|\mathbf{W}_{0}\right\|_{L^{p^{\prime}}} \leq R^{n / p^{\prime}}
$$

Moreover, if $i=1$ we have $\operatorname{div} \mathbf{V}_{0} \in L^{p}$,

$$
\left\|\operatorname{div} \mathbf{V}_{0}\right\|_{L^{p}}=\left(\int\left|\frac{1}{R}\left(\operatorname{div} \mathbf{V}_{1}\right)\left(\frac{x-x_{0}}{R}\right)\right|^{p} d x\right)^{1 / p}=R^{n / p-1}\left\|\operatorname{div} \mathbf{V}_{1}\right\|_{L^{p}} \leq R^{n / p-1},
$$

and

$$
\operatorname{curl} \mathbf{W}_{0}=R^{-1}\left(\operatorname{curl} \mathbf{W}_{1}\right)\left(R^{-1}\left(x-x_{0}\right)\right)=0,
$$

while if $i=2$ we have $\operatorname{div} \mathbf{V}_{0}=0$ and, for all $j, k \in\{1, \ldots, n\}$,

$$
\left\|\left(\operatorname{curl} \mathbf{W}_{0}\right)_{j k}\right\|_{L^{p}}=\left(\int\left|\frac{1}{R}\left(\left(\operatorname{curl} \mathbf{W}_{2}\right)_{j k}\right)\left(\frac{x-x_{0}}{R}\right)\right|^{p^{\prime}} d x\right)^{1 / p^{\prime}} \leq R^{n / p^{\prime}-1} .
$$

Letting $\mathbf{V}=R^{-n / p} \mathbf{V}_{0}, \mathbf{W}=R^{-n / p^{\prime}} \mathbf{W}_{0}$ and using the fact that $R \geq 1$, we get vector fields satisfying either (2.1) in the first case, or (2.2) in the second case, and

$$
\begin{aligned}
\int g \mathbf{V} \cdot \mathbf{W} & =R^{-n} \int \tilde{g}\left(R^{-1}\left(x-x_{0}\right)\right) \mathbf{V}_{i}\left(R^{-1}\left(x-x_{0}\right)\right) \cdot \mathbf{W}_{i}\left(R^{-1}\left(x-x_{0}\right)\right) d x \\
& =\int \tilde{g} \mathbf{V}_{i} \cdot \mathbf{W}_{i} .
\end{aligned}
$$

Taking the supremum over all such $\mathbf{V}_{i}, \mathbf{W}_{i}$, and using estimate (2.13) for the unit ball, we get the same inequality for the ball $B$. This concludes the proof of the theorem.

Remarks.

(1) All vector fields constructed in the proof are smooth with compact support, so the suprema on the right-hand side of (2.3) and (2.4) can be restricted to dot products of such vector fields.

(2) As pointed out, the proof of Case I is independent of the radius of the ball and therefore we have generalized the result (1.4) from CLMS to $p \neq 2$. Such a generalization and the resulting decomposition of $H^{1}\left(\mathbb{R}^{n}\right)$ in terms of (smooth) div-curl atoms is stated in BIJZ (Proposition 2.2) without proof.

\section{THE DECOMPOSITION THEOREM FOR $H^{1}$}

Since $\mathcal{D} \mathcal{C}_{1,0}^{p}$ (respectively $\mathcal{D} \mathcal{C}_{0,1}^{p}$ ) is a bounded symmetric subset of $h^{1}\left(\mathbb{R}^{n}\right)$, we can use Lemmas III.1 and III.2 in CLMS, the duality of bmo and $h^{1}$ [Go, and Theorem 2.2 to obtain the following decomposition of functions in $h^{1}$ in terms of the appropriate "div-curl atoms": 
Theorem 3.1. For a function $f$ in $h^{1}\left(\mathbb{R}^{n}\right), 1<p<\infty$, there exists a sequence $\left\{\lambda_{k}\right\} \in \ell_{1}$ such that

$$
f=\sum_{k=1}^{\infty} \lambda_{k} f_{k}, \quad f_{k} \in \mathcal{D C} \mathcal{C}_{1,0}^{p} \quad \text { for all } k \geq 1
$$

Such a decomposition also holds with $f_{k} \in \mathcal{D C}_{0,1}^{p}$ for all $k$.

\section{REFERENCES}

[BIJZ] Bonami, A., Iwaniec, T., Jones, P., Zinsmeister, M.: On the product of functions in BMO and $H^{1}$, Ann. Inst. Fourier (Grenoble) 57 (2007), pp. 1405-1439. MR2364134 (2009d:42054)

[CLMS] Coifman, R., Lions, P.-L., Meyer,Y., Semmes, S.: Compensated compactness and Hardy spaces, J. Math. Pures Appl. 72 (1993), pp. 247-286. MR1225511 (95d:46033)

[D] Dafni, G.: Nonhomogeneous div-curl lemmas and local Hardy spaces, Adv. Differential Equations 10 (2005), pp. 505-526. MR.2134048(2006a:42030)

[DL] Duvaut, G., Lions, J.-L.: Inequalities in Mechanics and Physics, Translated from the French by C. W. John, Springer-Verlag, Berlin-New York, 1976. MR0521262 (58:25191)

[FS] Fefferman, C., Stein, E. M.: $H^{p}$ spaces of several variables, Acta Math. 129 (1972), pp. 137-193. MR0447953 (56:6263)

[GR] Girault, V., Raviart, P.-A.: Finite Element Methods for Navier-Stokes Equations: Theory and Algorithms, Springer-Verlag, Berlin, 1980. MR851383 (88b:65129)

[Go] Goldberg, D.: A local version of real Hardy spaces, Duke Math. J. 46 (1979), pp. 27-42. MR.523600 (80h:46052)

[Mu F. Murat: Compacité par compensation, Ann. Scuola Norm. Sup. Pisa Cl. Sci. (4), Vol. 5 (1978), pp. 489-507. MR506997 (80h:46043a)

[Ne] J. Nečas: Sur les normes équivalentes dans $W_{p}^{K}(\Omega)$ et sur la coercitivité des formes formellement positives, in "Équations aux Dérivées partielles", Sémin. Math. Sup. 1965, Presse Univ. Montréal, Montréal, 1966.

Department of Mathematics, Georgetown University, Washington, DC 20057

E-mail address: chang@georgetown.edu

Department of Mathematics and Statistics, Concordia University, Montreal, QueBEC, H3G 1M8, CANADA

E-mail address: gdafni@mathstat.concordia.ca

Department of Mathematics and Informatics, Trine University, Angola, Indiana 46703

E-mail address: yueh@trine.edu 